Portland State University

PDXScholar

Electrical and Computer Engineering Faculty

Publications and Presentations

Electrical and Computer Engineering

$1-1-1993$

\title{
Ultrashort-Pulse Propagation in Dye Laser Amplifiers
}

Shuanghua Jiang

Portland State University

Lee W. Casperson

Portland State University

Follow this and additional works at: https://pdxscholar.library.pdx.edu/ece_fac

Part of the Electrical and Computer Engineering Commons

Let us know how access to this document benefits you.

\section{Citation Details}

Jiang, S., \& Casperson, L. W. (1993). Ultrashort-pulse propagation in dye laser amplifiers. Journal Of Applied Physics, 73(2), 530.

This Article is brought to you for free and open access. It has been accepted for inclusion in Electrical and Computer Engineering Faculty Publications and Presentations by an authorized administrator of PDXScholar. Please contact us if we can make this document more accessible: pdxscholar@pdx.edu. 


\title{
Ultrashort-pulse propagation in dye laser amplifiers
}

\author{
Shuanghua Jiang and Lee W. Casperson \\ Department of Electrical Engineering, Portland State University, Portland, Oregon 97207-0751
}

(Received 17 January 1992; accepted for publication 5 October 1992)

\begin{abstract}
A semiclassical model is presented for ultrashort-pulse dye laser amplifiers. The model permits a discussion of important aspects of short-pulse amplification such as pulse shape, pulse energy, and gain saturation. Coherence effects are found to be important in characterizing the evolution of amplified pulses in the subpicosecond and femtosecond regimes. The results are compared with those obtained using the corresponding rate equation model.
\end{abstract}

\section{INTRODUCTION}

Interest in ultrashort-pulse amplification has grown rapidly during the past few years, and because of their high gain and broad gain spectrum, liquid-dye-based laser amplifiers are usually considered to be the most attractive amplifying media. Amplification of pulses as short as $26 \mathrm{fs}$ has been reported by Knox in dye lasers. ${ }^{1}$ Many different pump sources have been employed in dye laser amplifiers including $\mathrm{cw}$ YAG lasers, ${ }^{2-5}$ argon-ion lasers, ${ }^{6,7}$ copper vapor lasers, ${ }^{8}$ and $\mathrm{XeCl}$ excimer lasers. ${ }^{9,10}$ Some of these systems were used to obtain pulses at high repetition rates, while others emphasized high peak powers. However, they all try to maintain pulse widths of less than $100 \mathrm{fs}$. In spite of the rapid development of experimental techniques for ultrashort optical pulse amplification, the theory of this subject seems to have lagged behind. To date, only a few theoretical studies on picosecond or subpicosecond dye laser pulse amplification have been reported. ${ }^{11-15}$ These rate equation models have explained many aspects of dye laser amplifier performance in the picosecond regime. However, they meet serious difficulties as the optical pulses go to the subpicosecond and femtosecond regime.

First, as these models usually originate from basic rate equation concepts, they may not consider the molecular vibrational relaxation time, which is on the order of a picosecond. This relaxation time has already been shown to be of importance in interpreting the evolution of picosecond laser pulses. ${ }^{16}$ In the subpicosecond or femtosecond regime, the effects of this relaxation time are expected to be even more substantial.

Second, in these models the coherence time or the dephasing time of the dye molecules of the amplifying medium was not taken into account. Depending on solvents, spectral positions and sample deterioration, this time has been found to lie in the range of $20 \mathrm{fs}$ to $2 \mathrm{ps},{ }^{16}$ which might be unimportant for picosecond pulses but not for femtosecond pulses. Rate equation models describe the light-matter interaction based on the assumption that the polarization varies instantaneously with the field, or in other words that the coherence time is zero. Such an assumption may be valid when the amplified pulses are much longer than the coherence time, i.e., the signal field varies much slower than the dephasing time. However, when the signal pulse width approaches the coherence time, rate equation models fail to explain the matter-light interaction adequately. Coherence time effects have been studied in excimer amplifying media such as $\mathrm{KrF}$ and $\mathrm{XeCl} \cdot{ }^{17-20} \mathrm{Sig}$ nificant differences, especially in the amplified pulse temporal evolution, were found between the results obtained with and without consideration of the coherence time. As dye laser media have a much different gain spectrum and energy structure, those models cannot be used to describe the complex nonlinear processes that take place inside dye laser amplifiers.

Third, in order to characterize the dye medium, the molecular orientational distribution must be included in the model. With parallel pump and signal polarizations and modest pumping levels, a unidirectional orientational distribution can provide qualitative insight into some aspects of dye laser amplification. However, inclusion of the more realistic isotropic distribution has been shown to be necessary for quantitative interpretations or predictions concerning synchronously pumped mode-locked dye laser oscillators. ${ }^{16}$

Starting from the model derived in Ref. 21, which was based on the density matrix equations and Maxwell's equations, we have studied in detail the dynamic behavior of ultrashort pulses propagating in a dye laser amplifier. A general semiclassical model for dye laser amplifiers is derived in Sec. II. Numerical results and comparison of the general semiclassical model with the corresponding rate equation model and the unidirectional molecular distribution model are given in Sec. III.

\section{THEORY}

\section{A. Semiclassical model}

The energy-level model used in the present study is the four-level system shown in Fig. 1. This is the same model used previously in studies of coherence effects and pump polarization effects in synchronously pumped mode-locked dye lasers. ${ }^{16,21}$ The pump absorption occurs between level 0 and level 3 , while the signal stimulated emission takes place between levels 2 and 1. The molecules in level 3 decay nonradiatively to level 2 with a vibrational relaxation time of $\tau_{3}$ while molccules in level 1 have a vibrational relaxation time of $\tau_{1}$. The spontaneous decay time for the laser transition is represented by $\tau_{2}$. Setting up the density matrix equations for the dye medium and combining them with Maxwell's equations for the electric field in 


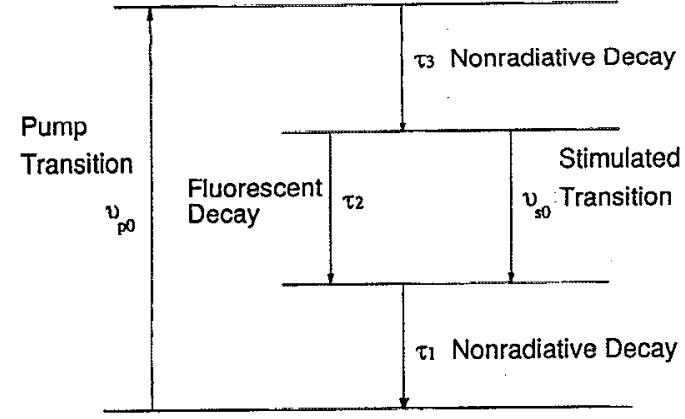

FIG. 1. Energy-level model used in the dye laser analysis.

the medium leads to a set of nonlinear coupled equations, which were derived as Eqs. (23)-(26) in Ref. 21:

$$
\begin{aligned}
\frac{\partial D}{\partial t}= & -\frac{1}{\tau_{2}}\left\{\left(1+\frac{\tau_{2}}{2 \tau_{1}}\right) D+\left(1-\frac{\tau_{2}}{2 \tau_{1}}\right) M+2 Q A \chi\right. \\
& \left.-P\left[\chi^{2}\left(1-\frac{3 \sin ^{2} \alpha}{2}\right)+\frac{\sin ^{2} \alpha}{2}\right]\right\}, \\
\frac{\partial M}{\partial t}= & -\frac{1}{\tau_{2}}\left\{-\frac{\tau_{2}}{2 \tau_{1}} D+\frac{\tau_{2}}{2 \tau_{1}} M\right. \\
& \left.-P\left[\chi^{2}\left(1-\frac{3 \sin ^{2} \alpha}{2}\right)+\frac{\sin ^{2} \alpha}{2}\right]\right\}, \\
\frac{\partial Q}{\partial t}= & -\frac{1}{T_{s}}(Q-A D \chi), \\
\frac{\partial A}{\partial z}+\frac{1}{v} & \frac{\partial A}{\partial t}=-\frac{\gamma_{s}}{2}\left(A-S_{0}^{1} Q \chi d \chi\right) .
\end{aligned}
$$

In these equations $T_{s}$ is the coherence time of the dye molecules, $\gamma_{s}$ is a distributed loss coefficient, and $v=(\mu \epsilon)^{-1 / 2}$ is the velocity of the signal field. There are four unknowns associated with this set of coupled equations: $D$ is a normalized population difference, $M$ is a normalized population sum, $Q$ is a normalized polarization, and $A$ is a normalized signal electric field. $P$ is a normalized pump rate which in this form of the equations includes the relaxation decay from level 3 to level 2 . These four unknowns and the pump rate are related to the fundamental quantities by

$$
\begin{aligned}
D(\theta, z, t)= & \frac{\mu \omega_{s}^{2} N T_{s}\left|\mu_{s}\right|^{2}}{k_{s} \hbar \gamma_{s}} \frac{\chi^{2}\left(2-3 \sin ^{2} \alpha\right)+\sin ^{2} \alpha}{2 X^{2}} \\
& \times\left(\rho_{22}-\rho_{11}\right), \\
M(\theta, z, t)= & \frac{\mu \omega_{s}^{2} N T_{s}\left|\mu_{s}\right|^{2}}{k_{s} \hbar \gamma_{s}} \frac{\chi^{2}\left(2-3 \sin ^{2} \alpha\right)+\sin ^{2} \alpha}{2 X^{2}} \\
& \times\left(\rho_{22}+\rho_{11}\right),
\end{aligned}
$$
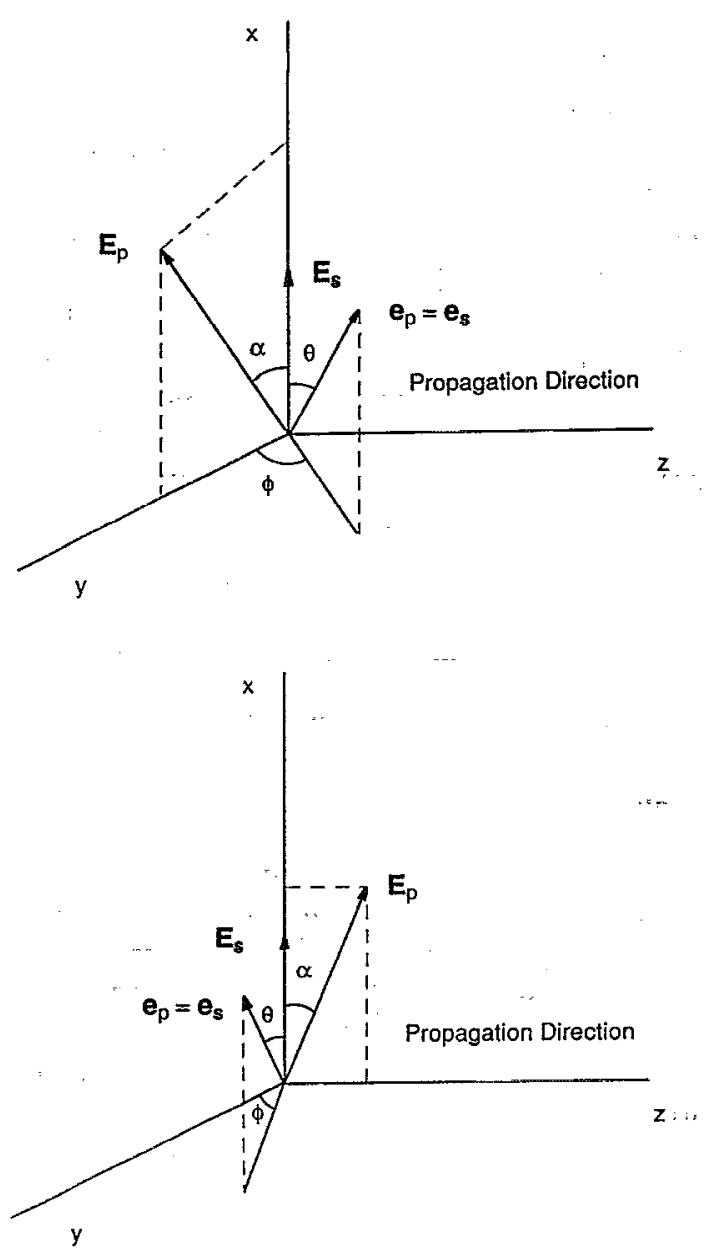

FIG. 2. Geometry of pump and signal fields and molecular dipole moments: (a) longitudinal pump; and (b) transverse pump.

$$
\begin{gathered}
Q(\theta, z, t)=\frac{\mu \omega_{s}^{2} N T_{s}\left|\mu_{s}\right|^{2}\left(2 \tau_{2} T_{s}\right)^{1 / 2}}{k_{s} \hbar \gamma_{s}} \\
\times \frac{\chi^{2}\left(2-3 \sin ^{2} \alpha\right)+\sin ^{2} \alpha}{2 X^{2}} \eta_{s}^{\prime}, \\
A(z, t)=\frac{\left|\mu_{s}\right|}{\hbar}\left(\frac{\tau_{2} T}{2}\right)^{1 / 2} E_{s}^{\prime}, \\
P=\frac{\mu \omega_{s}^{2} N T_{s}\left|\mu_{s}\right|^{2}}{k_{s} \hbar \gamma_{s}} \frac{\tau_{2} T_{p}\left|\mu_{p}\right|^{2} E_{p}^{\prime 2}}{2 \hbar^{2}},
\end{gathered}
$$

where $E_{p}^{\prime}$ and $E_{s}^{\prime}$ are, respectively, the magnitudes of the pump and signal electric fields, $\chi=\cos \theta$ and the variable $\theta$ measures the angle of a class of signal dipoles with respect to the signal field polarization, the angle $\alpha$ measures the misalignment between the pump field and the signal field, and $\phi$ measures the orientation of the dipoles around the $x$ axis as shown in Fig. 2. Also, $\rho_{11}$ and $\rho_{22}$ are the density matrix elements representing the populations of the lower and upper laser levels, and $\left|\mu_{p}\right|$ and $\left|\mu_{s}\right|$ are the magnitudes of the pump and signal dipole moments, respectively. The parameter $\eta_{s}^{\prime}$ is related to the slowly varying magnitude of the off-diagonal density matrix elements associated with the signal transition as follows: ${ }^{16}$ 


$$
\eta_{s}^{\prime}=-i \rho_{21}\left|\mu_{s}\right|
$$

where $i$ is the imaginary number unit, and $\rho_{21}$ is the offdiagonal element associated with the signal transition. The angular frequency of the signal transition $\omega_{s}$, propagation constant $k_{s}=\omega_{s}(\mu \epsilon)^{1 / 2}$, and the dye molecule concentration $N$, are also employed in the above equations.

Although Eqs. (1)-(4) were derived for the longitudinal pumping arrangement, they are also valid for transverse pumping arrangements (see Appendix). $X$ is an orientation factor related to the misalignment of the pump and signal field polarizations. For the longitudinal pumping as introduced in Ref. $21, X$ is

$$
X=\chi \cos \alpha+\left(1-\chi^{2}\right)^{1 / 2} \sin \alpha \cos \phi
$$

For the transverse pumping, $X[\mathrm{Eq} .(\mathrm{A} 7)]$ is

$$
X=\chi \cos \alpha+\left(1-\chi^{2}\right)^{1 / 2} \sin \alpha \sin \phi .
$$

\section{B. Simplifications}

In order to have the highest interaction efficiency, the pump field needs to have the same polarization as the signal field, which was previously demonstrated in detail in a synchronously pumped mode-locked dye laser system. ${ }^{21}$ In the present case, we will only consider the parallel alignment of the pump field and signal field. Therefore, one may substitute $\alpha=0$ into Eqs. (1)-(4), and it is helpful to introduce a new set of coordinate variables as

$$
\begin{aligned}
& \tau=t-(z / v), \\
& \xi=\gamma_{s} z .
\end{aligned}
$$

With some mathematical simplifications, one can express Eqs. (1) - (4) in terms of the new coordinate variables as

$$
\begin{aligned}
& \frac{\partial D}{\partial \tau}=-\frac{1}{\tau_{2}}\left[\left(1+\frac{\tau_{2}}{2 \tau_{1}}\right) D+\left(1-\frac{\tau_{2}}{2 \tau_{1}}\right) M+2 Q A \chi-P \chi^{2}\right] \\
& \frac{\partial M}{\partial \tau}=-\frac{1}{\tau_{2}}\left(-\frac{\tau_{2}}{2 \tau_{1}} D+\frac{\tau_{2}}{2 \tau_{1}} M-P \chi^{2}\right) \\
& \frac{\partial Q}{\partial \tau}=-\frac{1}{T_{s}}(Q-A D \chi) \\
& \frac{\partial A}{\partial \zeta}=-\frac{1}{2}\left(A-\int_{0}^{1} Q \chi d \chi\right)
\end{aligned}
$$

It is also helpful to introduce a pump threshold parameter $r$. Threshold may be defined as the condition when a steady-state signal sees zero distributed gain along an unsaturated amplifier. Therefore, from Eqs. (15)-(18), one finds

$$
\begin{aligned}
& 0=-\frac{1}{\tau_{2}}\left[\left(1+\frac{\tau_{2}}{2 \tau_{1}}\right) D+\left(1-\frac{\tau_{2}}{2 \tau_{1}}\right) M+2 Q A \chi-P \chi^{2}\right], \\
& 0=-\frac{1}{\tau_{2}}\left(-\frac{\tau_{2}}{2 \tau_{1}} D+\frac{\tau_{2}}{2 \tau_{1}} M-P \chi^{2}\right)
\end{aligned}
$$

$$
\begin{aligned}
& 0=-\left(1 / T_{s}\right)(Q-A D \chi), \\
& \frac{\partial A}{\partial z}=-\frac{\gamma_{s}}{2}\left(A-\int_{0}^{1} Q \chi d \chi\right) .
\end{aligned}
$$

Equation (21) has the solution

$$
Q=A D \chi,
$$

and Eqs. (19) and (20) have the solutions

$$
\begin{aligned}
& D=P \chi^{2}\left[1-\left(\tau_{1} / \tau_{2}\right)\right], \\
& M=D+\left(2 \tau_{1} / \tau_{2}\right) P \chi^{2}=P \chi^{2}\left[1+\left(\tau_{1} / \tau_{2}\right)\right] .
\end{aligned}
$$

Substitution of Eq. (23) into Eq. (22) using Eq. (24) leads to

$$
\begin{aligned}
\frac{\partial A}{\partial z} & =-\frac{\gamma_{s}}{2} A+\frac{1}{2} s_{0}^{1} A D \chi^{2} d \chi, \\
& =-\frac{\gamma_{s}}{2}\left[A-A P\left(1-\frac{\tau_{1}}{\tau_{2}}\right) s_{0}^{1} \chi^{4} d \chi\right] \\
& =-\frac{\gamma_{s}}{2} A\left[1-\frac{P}{5}\left(1-\frac{\tau_{1}}{\tau_{2}}\right)\right] .
\end{aligned}
$$

In terms of the dimensionless light intensity $I=A^{2}$, Eq. (26) can be expressed

$$
\frac{\partial I}{\partial z}=-\gamma_{s}\left[1-\frac{P}{5}\left(1-\frac{\tau_{1}}{\tau_{2}}\right)\right] I .
$$

As mentioned above, threshold may be defined as the condition when the distributed gain for the intensity is zero. Thus a threshold pump rate can be obtained from Eq. (27),

$$
P_{\text {threshold }}=5 \tau_{2} /\left(\tau_{2}-\tau_{1}\right) .
$$

The pump threshold parameter $r$ describes the degree of pumping above threshold. In terms of the threshold parameter, any pump rate $P$ can be expressed as

$$
P=r P_{\text {threshold }} \text {. }
$$

\section{NUMERICAL RESULTS}

By numerically solving Eqs. (15)-(18), one can obtain all of the parameters concerning ultrashort-pulse propagation in a dye laser amplifier. The physical situation of a pulse propagating in an amplifier is illustrated in the spacetime diagram of Fig. 3, which is similar to a pulse propagation description developed for maser amplifiers. ${ }^{22}$ It is seen that the new time coordinate $\tau$ remains constant for any part of the signal as it passes from the input to the output. For instance, the leading edge of a pulse may be characterized everywhere by $\tau=\tau_{0}$, whereas in real time it would pass the input at $t=t_{0}$ and the output at $t=t_{0}+L / v$ where $L$ is the amplifier length and $v$ is the speed of light.

The figure also suggests that the physical situation at a point $(\zeta, \tau)$ is determined by all interactions which happened earlier, i.e., from time $-\infty$ to $\tau$ and closer, i.e., from 0 to $\zeta$. Such a problem can not be specified unless two boundary conditions are set up. One is the initial condition which specifies the situation for values of space $0<\xi<L$ at 


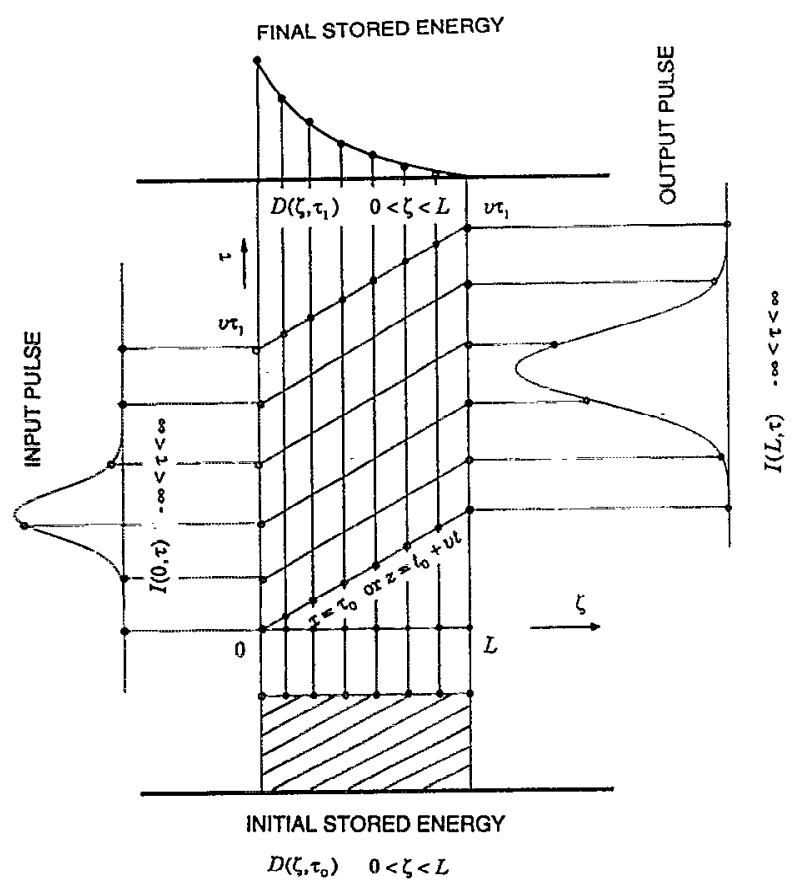

FIG. 3. Schematic diagram of a pulse propagating in a dye laser amplifier and the gain process by depletion of stored energy; also illustrated are the puise evolution in time $\tau$ and space $\zeta$.

initial time $\tau=-\infty$. The second boundary condition is the input condition which specifies the situation for time $-\infty$ $<\tau<\infty$ at the input $\zeta=0$. With these two boundary conditions, the equations permit a unique evaluation of the variables at any position $\zeta$ and time $\tau$.

The two boundary conditions for the present problem include the initial condition for the population difference $D$, population sum $M$, polarization $Q$, and signal field $A$ or intensity $I$. Since amplified spontaneous emission (ASE) is neglected here, there will be no electric field along the amplifier before the signal arrives. Also, assuming the population reaches steady state before the signal pulse arrives at the amplifier, the initial condition of $D$ and $M$ will have the same forms as those determined in Eqs. (24) and (25), which are

$$
D(\zeta, \tau=-\infty)=P(\zeta) \chi^{2}\left[1-\left(\tau_{1} / \tau_{2}\right)\right]
$$

for $0<\xi<L$ and zero anywhere else,

$M(\zeta, \tau=-\infty)=P(\zeta) \chi^{2}\left[1+\left(\tau_{1} / \tau_{2}\right)\right]$

for $0<\zeta<L$ and zero anywhere else.

The initial time here is defined as the moment just before the input pulse enters the amplifier. Since ASE is assumed to be negligible, the initial condition for the electric field is zero, as is the initial condition for the polarization $Q . P(\zeta)$ is the pump distribution. Although an arbitrary pump distribution can be employed in the model, a constant pump has been assumed for the present problem. This simplified case would be valid in a weakly absorbing medium as long as the pump pulse is longer than the longitudinal dimension of the amplifier cell in space, and longer than the signal pulse in time. These conditions would also be satisfied for most practical amplifier setups including transverse pump configurations.

The other condition describes the boundary of the normalized electric field at $\xi=0$, which is actually the input signal pulse

$A(\zeta=0, \tau)=\left\{W\left(\frac{2}{\Delta \tau_{i}}\right)\left(\frac{\ln 2}{\pi}\right)^{1 / 2} \exp \left[-\left(\frac{2 \tau}{\Delta \tau_{i}}\right) \ln 2\right]\right\}^{1 / 2}$,

where an intensity pulse with a Gaussian profile was assumed. $W$ is a normalized pulse energy density, and in this representation the units of $W$ are seconds. The boundary condition for the other quantities cannot be specified directly. However, after the electric field or the intensity of the pulse is specified, $D(\xi=0, \tau), M(\xi=0, \tau)$, and $Q(\xi$ $=0, \tau)$ can be resolved from Eqs. (15)-(17), which are one-dimensional, coupled first-order differential equations. A fourth-order Runge-Kutta method was used to obtain these numerical solutions.

The parameters related to Rhodamine $6 \mathrm{G}$ include the vibrational relaxation time $\tau_{1}=1 \mathrm{ps}$, the fluorescence decay time $\tau_{2}=5 \mathrm{~ns}$, and the coherence time $T_{s}=50 \mathrm{fs}$. The signal at arbitrary space and time $(\zeta, \tau)$ was computed as illustrated in Fig. 3. At any constant $\zeta$, the signal at time $\tau$ was determined by the population difference, population sum, and the polarization at that time and earlier. At any time, the signal at a point $\zeta$ was determined by the interaction before that position. A recursive formula for space and time was used for the entire time range and for the position along the amplifier cell.

\section{A. General results}

A normalized energy density of $W=1 \times 10^{-8} \mathrm{~s}$ was employed for the input pulse. In agreement with most experimental configurations, this input is well above the spontaneous emission level and leads to strong saturation in a gain length product of less than $g l=10$. The full width at half-maximum (FWHM) of the input pulse is chosen as $1 \mathrm{ps}, 100 \mathrm{fs}$, and $10 \mathrm{fs}$, and the threshold parameter is $1 \times 10^{4}$. With these parameters, solutions of Eqs. (15)(18) have been carried out, and the results are discussed in the following subsection.

\section{B. Rate equation approximation}

In the rate equation limit where the polarization varies simultaneously with the electric field, the coherence time $T_{s}$ can be treated as zero. As a result, Eq. (17) has the solution

$$
Q=A D \chi .
$$

If this solution is substituted into Eqs. (15), (16), and (18), one obtains

$$
\frac{\partial D}{\partial \tau}=-\frac{1}{\tau_{2}}\left[\left(1+\frac{\tau_{2}}{2 \tau_{1}}\right) D+\left(1-\frac{\tau_{2}}{2 \tau_{1}}\right) M+2 A^{2} \chi^{2}-P \chi^{2}\right],
$$



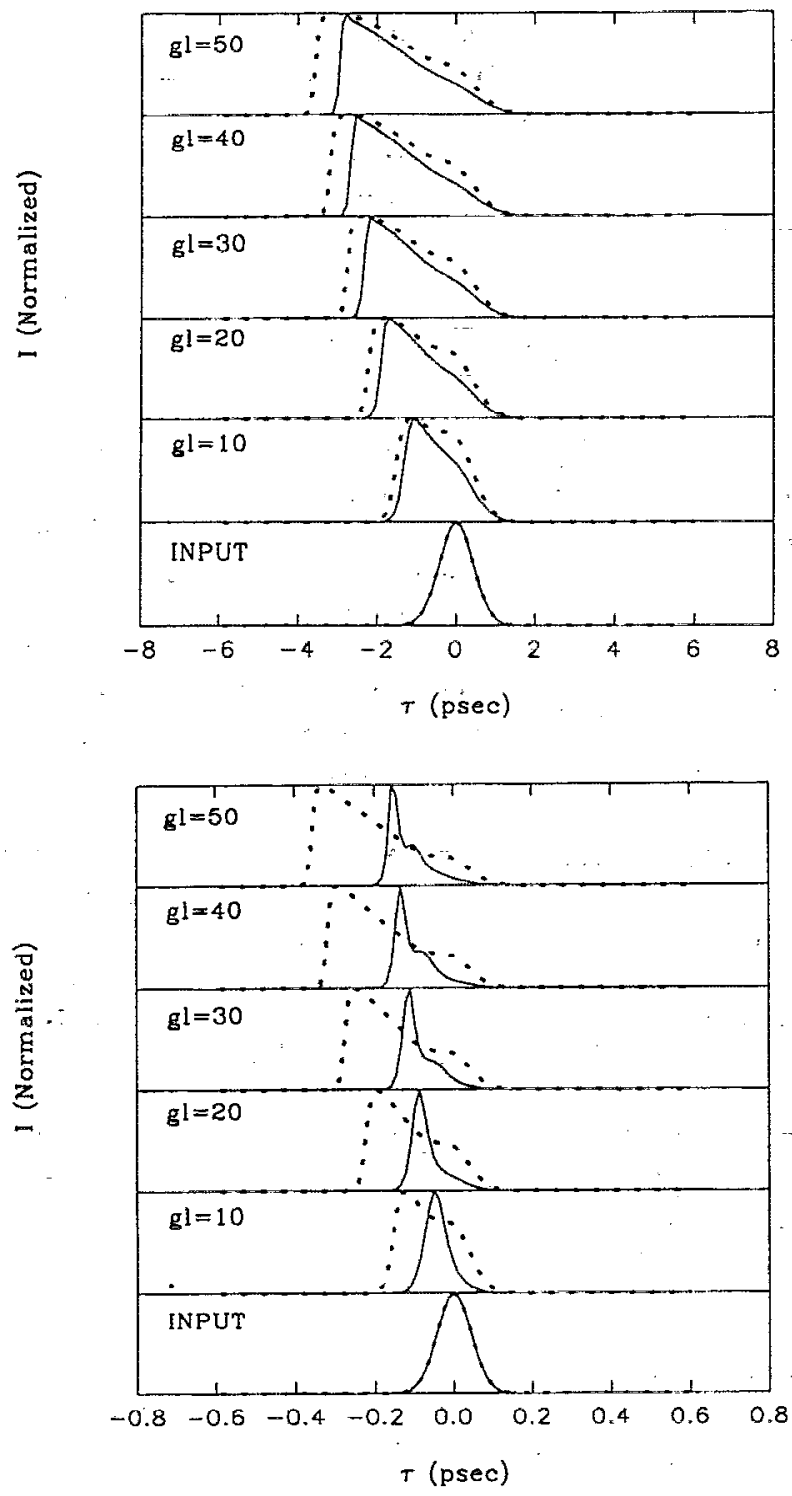

$$
\begin{aligned}
& \frac{\partial M}{\partial \tau}=-\frac{1}{\tau_{2}}\left(-\frac{\tau_{2}}{2 \tau_{1}} D+\frac{\tau_{2}}{2 \tau_{1}} M-P \chi^{2}\right), \\
& \frac{\partial A}{\partial \zeta}=-\frac{A}{2}\left(1-\int_{0}^{1} D \chi^{2} d \chi\right) .
\end{aligned}
$$

In this limit, a set of three nonlinear coupled differential equations, instead of four as in the above sections, is employed to describe the light-matter interaction in a dye laser amplifier. Figure 4 illustrates the pulse intensity (normalized to its peak value) as a function of time for both the general semiclassical model and the rate equation approximation at small signal gain lengths $g l$ of $10,20,30,40$, and 50. Parts (a), (b), and (c) correspond to input pulse widths of $1 \mathrm{ps}, 100 \mathrm{fs}$, and $10 \mathrm{fs}$, respectively. The solid lines represent the general semiclassical model and the dotted lines represent the rate equation model. Different time scales should be noticed in these figures. The time corresponding to the peak of the input pulses for all of the cases is at zero. As the input pulse propagates in the amplifier, the leading edge of the pulse experiences larger gain than

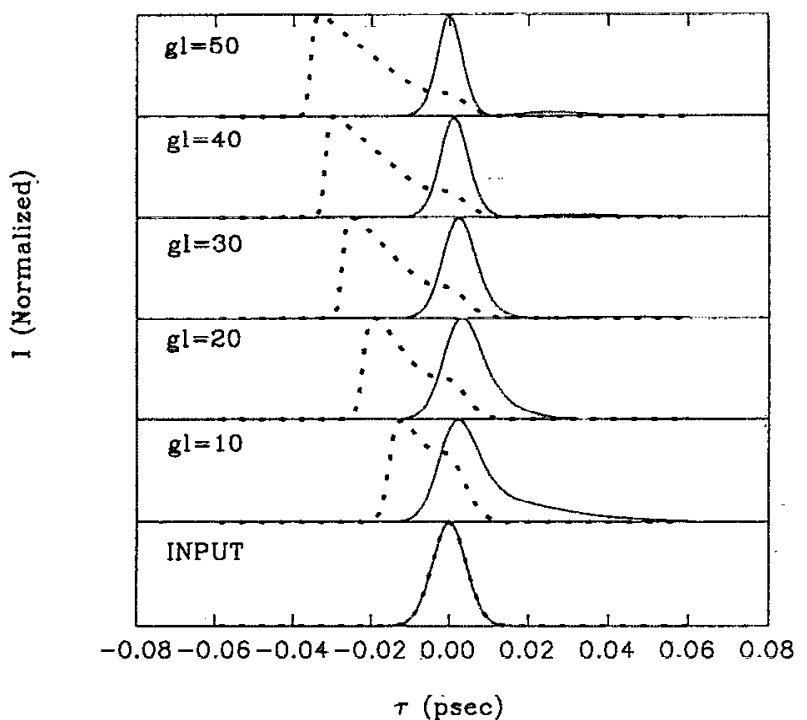

FIG. 4. Normalized intensity temporal profiles of amplified pulses with initial small signal gain-length product from 10 to 50 based on the gencral semiclassical model (solid lines) given in Eqs. (15)-(18) and rate equation model (dotted lines) given in Eqs. (34)-(36). A normalized input pulse energy density of $1 \times 10^{-8} \mathrm{~s}$ and a threshold parameter of $10^{4}$ were employed. The input pulse widths are (a) $1 \times 10^{-12} \mathrm{~s}$, (b) $1 \times 10^{-13} \mathrm{~s}$, and (c) $1 \times 10^{-14} \mathrm{~s}$. Different time scales should be noticed in cases (a), (b), and (c).

the tail due to the gain saturation; the peak of the pulse travels faster than the speed of light. The sharpening of the leading edge and the pulse broadening can be seen clearly, which are some of the well-known characteristics of pulse propagation inside an amplifier predicted by rate equation models as seen in case (a). It is seen that the semiclassical model and the rate equation model predict similar intensity temporal profiles in this range where the signal pulse width is long compared to the coherence time. When the pulse width becomes shorter as in case (b), however, the difference appears more obvious; and the rate equation model begins to show some inaccuracies. The pulse width predicted by the semiclassical model is much shorter than that predicted by rate equations. The pulse delay time is also different but the peak is still moving forward with respect to the speed of light. When the input pulse becomes much shorter, as in case (c), the peak may even move slower than the speed of light and the pulse width may become smaller as the pulse propagates along the amplifier.

It can be seen from the right-hand side of the field 
wave equation of Eq. (18) that the field cannot be separated out for the general semiclassical model, and the conventional idea of field or intensity gain has little meaning. The population difference between the laser transition levels is of more significance. The curves in Fig. 5 show the temporal behavior of the integrated (over the entire solid angle) population difference, and time scales for parts (a), (b), and (c) are the same as those in Fig. 4. The solid lines represent the semiclassical model, and the dotted lines represent the rate equation model. The population difference is always positive in the rate equation model but can be negative in some time regimes in the semiclassical model due to the coherence time effects. When the pulse width becomes comparable to the coherence time, the induced polarization can not respond instantaneously to changes in the population inversion and electric field, but is delayed due to the finite coherence time. As a result, the pulse can even be amplified when the population difference is negative. When the polarization changes from positive to negative or changes from a larger to a smaller value, the amplifier may turn into an absorber. In this case, the amplifying medium behaves like a saturable absorber which quenches the tail part of the pulse. Thus the pulse width can be shortened as the pulse is propagating in the amplifier.

The integrated energy gain versus the small signal gain-length product $g l$ is plotted in Fig. 6(a) for the general semiclassical model (a) and in Fig. 6(b) for the rate equation model. Even though the temporal behavior of the amplified pulses may vary dramatically, the energy gain curves predicted by both models for pulses with different pulse widths are almost the same. The same energies and shorter pulses predicted by the semiclassical model imply higher peak intensities. For the input pulse we have used, the energy gain is strongly saturated by about $g l=10$ for all cases.

\section{Unidirectional molecular distribution}

For quantitative investigation of dye laser amplifiers, the isotropic molecular distribution is required as discussed in the previous subsection above. Under modest pump power, however, the unidirectional molecular distribution is a common way to simplify and qualitatively describe the interaction in dye lasers as well as to use less computer time. Even though the unidirectional distribution model, or an averaged absorption and stimulated emission cross section, is employed sometimes, such models are not as general and rigorous as the isotropic distribution model. In this subsection, we will compare the unidirectional distribution model with the isotropic distribution model and clarify the inaccuracy of the unidirectional distribution model.

By simplifying Eqs. (15)-(18), one can obtain a set of equations for the unidirectional case,

$$
\frac{\partial D}{\partial \tau}=-\frac{1}{\tau_{2}}\left[\left(1+\frac{\tau_{2}}{2 \tau_{1}}\right) D+\left(1-\frac{\tau_{2}}{2 \tau_{1}}\right) M+2 Q A-P\right],
$$

$$
\begin{aligned}
& \frac{\partial M}{\partial \tau}=-\frac{1}{\tau_{2}}\left(-\frac{\tau_{2}}{2 \tau_{1}} D+\frac{\tau_{2}}{2 \tau_{1}} M-P\right), \\
& \frac{\partial Q}{\partial \tau}=-\frac{1}{T_{s}}(Q-A D), \\
& \frac{\partial A}{\partial \xi}=-\frac{1}{2}(A-Q) .
\end{aligned}
$$

The parameters used here were the same as those in Secs. III A and III B; however, the threshold pump rate required to have zero distributed gain for the signal intensity is changed to

$$
P_{\text {threshold }}=\tau_{2} /\left(\tau_{2}-\tau_{1}\right) \text {. }
$$

The threshold pump in the unidirectional case is a fifth of that for the isotropic distribution. This is reasonable since in the undirectional case all the molecular dipoles are aligned in the direction of the pump and signal fields, and all the dipoles contribute to the interaction. The pump rate can be expressed in terms of $r$ as

$$
P=r P_{\text {threshold }}
$$

The results of a unidirectional molecular distribution model for the pulse propagation in a dye laser amplifier are compared with those of the general semiclassical model, which employed the isotropic molecular distribution, in the following figures. Figure 7 shows the intensity profiles as a function of time for input pulse widths of $1 \mathrm{ps}, 100 \mathrm{fs}$, and $10 \mathrm{fs}$. It is found that the pulses in the unidirectional distribution case are qualitatively similar to those in the general semiclassical model. The echo pulses in the unidirectional case are farther apart and hold more energy. Intuitively, the unidirectional distribution should lead to larger intensities since all the dipoles are aligned with the signal and pump fields. However, it should be noticed that to reach the same level above threshold, the pump for an isotropic distribution has to be five times as large as for a unidirectional distribution. The pump rate we have employed is normalized to $P_{\text {threshold }}$, and thus the isotropic distribution can give higher intensity than the unidirectional distribution.

Figure 8 shows the population difference for the isotropic distribution and the unidirectional distribution. It is found that coherence time effects in the unidirectional case are stronger. The pulse energy gains are still similar for both cases as illustrated in Fig. 9.

\section{CONCLUSION}

A theoretical model has been developed for the propagation of ultrashort pulses in dye laser amplifiers. In comparison with the results from rate equation models, one finds that a semiclassical model including the finite coherence time, isotropic molecular distribution, and fast vibrational relaxation time should be much more accurate in characterizing nonlinear processes in the amplifier. Although some pulse amplification systems employ a saturable absorber together with the amplifier to compress the pulses, it is clear from this investigation that such ap- 

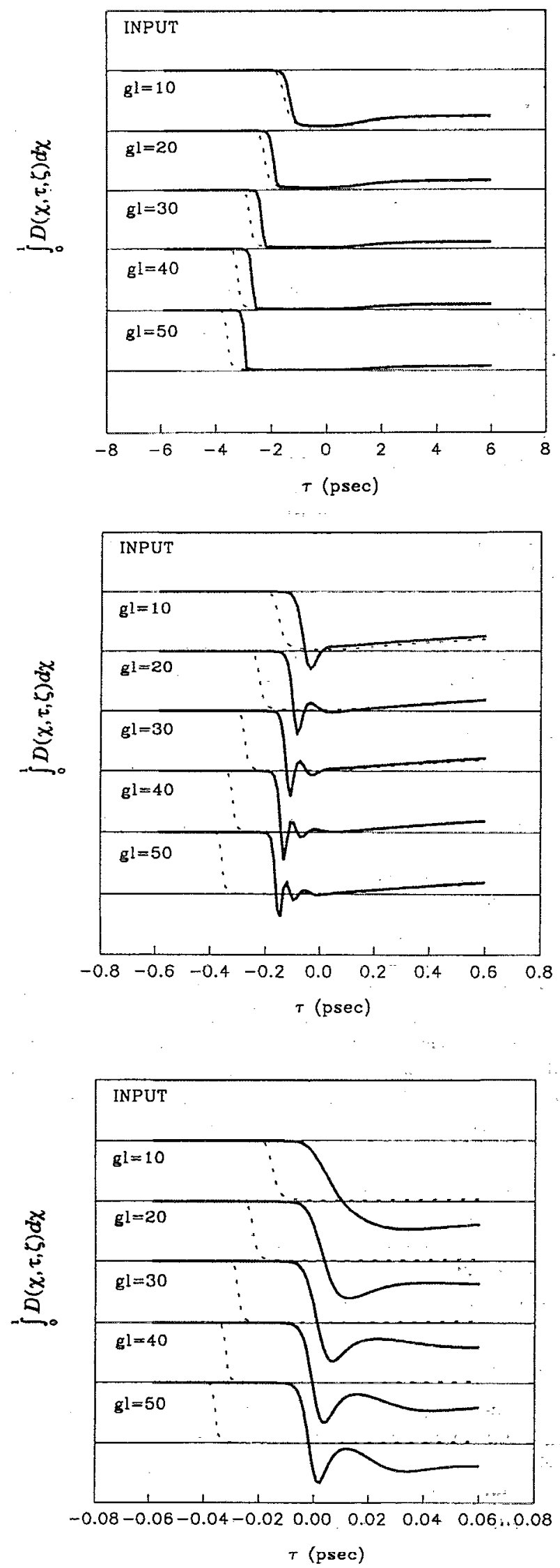

FIG. 5. Normalized integrated (over the entire solid angle) population differences as a function of time. Solid lines are the general semiclassical model, and dotted lines are the rate equation model. The input pulse widths are (a) $1 \times 10^{-12} \mathrm{~s}$, (b) $1 \times 10^{-13}$, and (c) $1 \times 10^{-14} \mathrm{~s}$.
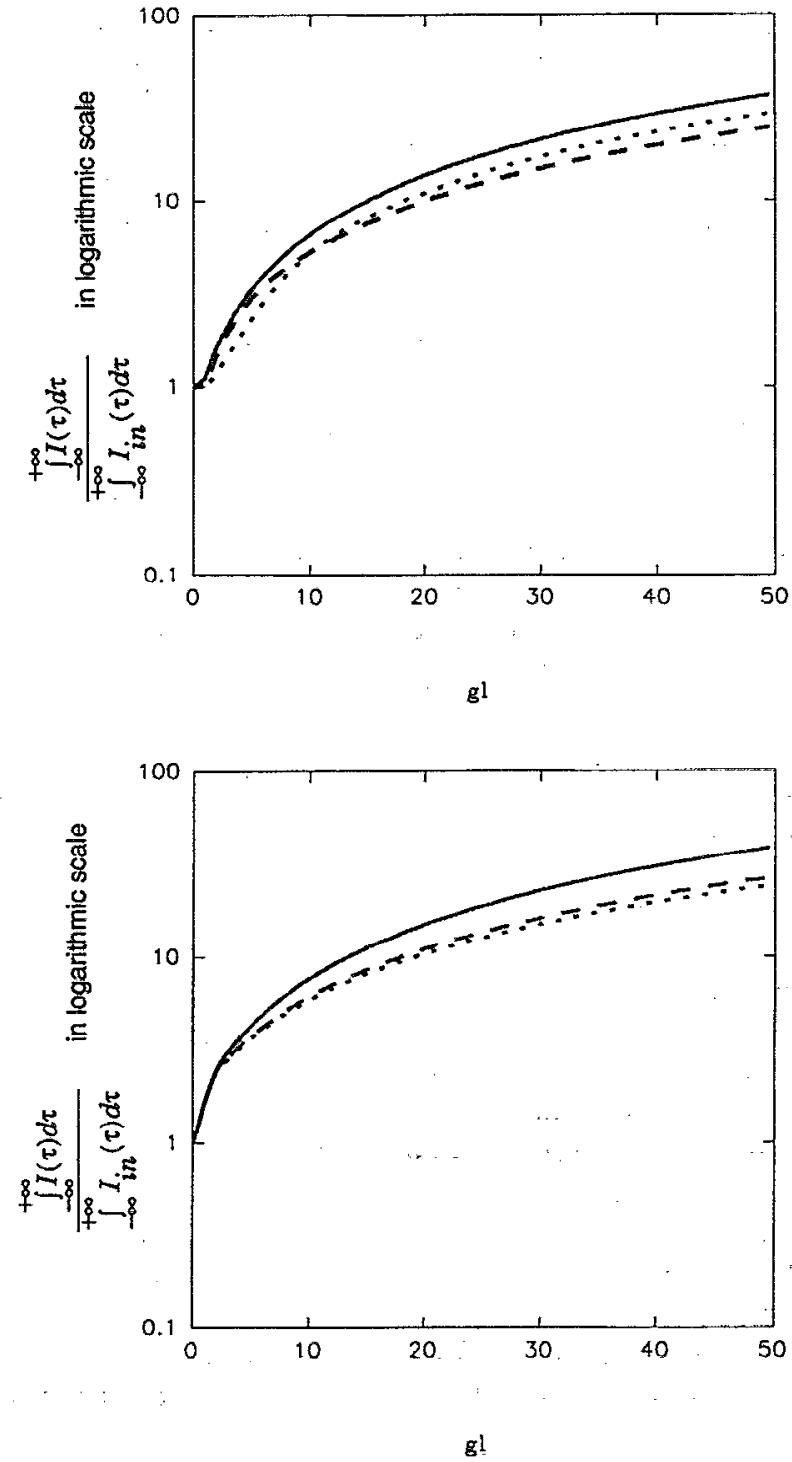

FIG. 6. Integrated pulse energy gain based on (a) the general semiclassical model and (b) the rate equation model as a function of small signal gain-length product for input pulse widths of $1 \times 10^{-12} \mathrm{~s}$ ( solid lines), $1 \times 10^{-13} \mathrm{~s}$ (dashed lines), and $1 \times 10^{-14} \mathrm{~s}$ (dotted lines). Other parameters are the same as in Fig. 4.

proaches should be preceded by more detailed studies of the amplifier itself. Such studies are especially needed for subpicosecond and femtosecond pulses where the inaccuracies of the rate equation models are particularly conspicuous. The more accurate models will provide the necessary accurate pulse information on parameters such as pulse shape, pulse energy, etc., for subsequent amplifier or absorber stages. Due to the self-compression arising from the coherence time effects in some ranges, the pulse may be directly amplified to high energy without serious pulse broadening and distortion. It should also be noted that models similar to those developed here may be applied to many types of absorbers.

Although a unidirectional molecular distribution assumption could simplify the investigation of the ultrashort pulse amplification in the dye laser amplifier, such a model 

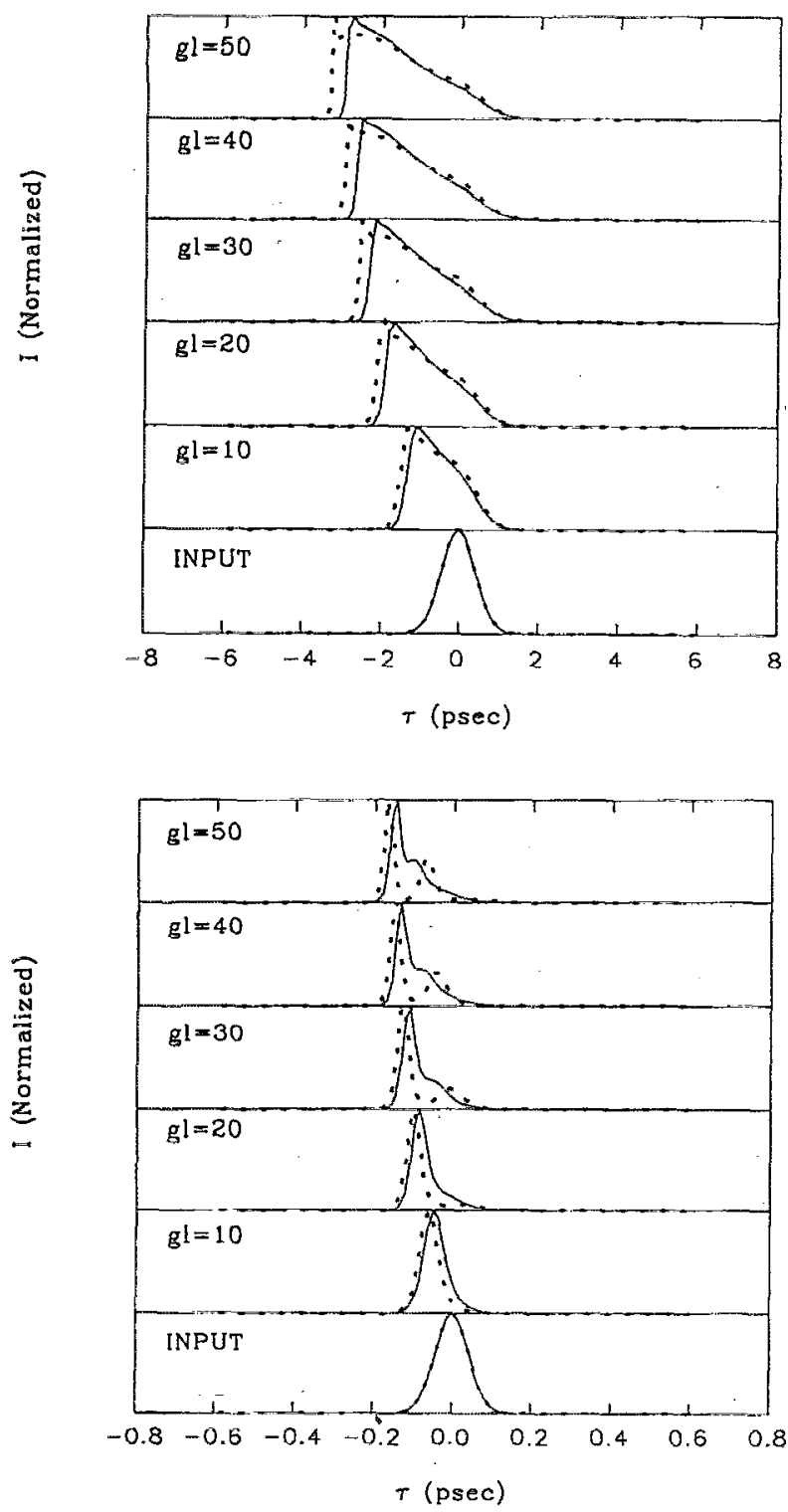

is somewhat unrealistic. Qualitatively, a unidirectional molecular distribution model could describe the amplified pulse shape, pulse energy, etc. Quantitatively, however, it could overestimate the coherence effects. To obtain quantitative results, the more general and rigorous isotropic molecular distribution model as developed in Eqs. (15)(18) should be utilized.

The semiclassical model for the ultrashort pulse dye laser amplifier developed here overcomes the limit of the rate equation approximation of zero coherence time. It is, however, still under the approximation of slowly varying electric-field amplitude. Such a treatment is valid as long as the signal transient time is much longer than an optical cycle, which is about 2 fs for a signal at a wavelength of $630 \mathrm{~nm}$. Moreover, due to the nonlinearity of the dye solvents, other nonlinear effects such as self-phase modulation and group velocity dispersion should also be considered at very high intensities or in long amplifier cells.

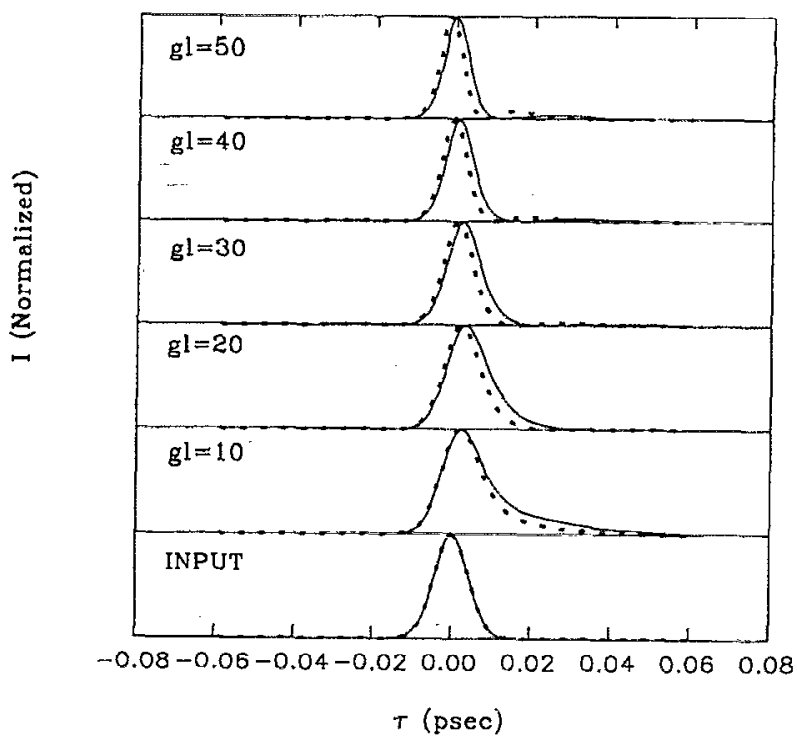

FIG. 7. Normalized intensity temporal profiles of amplified pulses with initial small signal gain-length product from 10 to 50 based on the general (isotropic molecular distribution) semiclassical model (solid lines) given in Eqs. (15)-(18) and the unidirectional molecular distribution model (dotted lincs) given in Eqs. (37)-(40). Parameters are the same as in Fig. 4.

\section{ACKNOWLEDGMENTS}

This work was supported in part by the National Science Foundation under Grant No. ECS-9014481 and by Tektronix, Inc.

\section{APPENDIX}

The laser model developed in Ref. 21 and summarized in Eqs. (1)-(4) was based on the assumption that the pump and signal fields propagate in the same longitudinal direction, as shown in Fig. 2(a). However, it is also common in dye laser amplifiers to have the pump field incident transverse to the direction of the signal propagation. ${ }^{15}$ The purpose of this appendix is to show that the basic laser equations for transverse pumping can be written in the same form as Eqs. (1)-(4).

For the transverse pump arrangement as shown in Fig. 2(b), the signal field $\mathbf{E}_{s}^{\prime}$ is parallel to the $x$ axis, and the pump field $\mathbf{E}_{p}^{\prime}$ is linearly polarized in the $x z$ plane and 

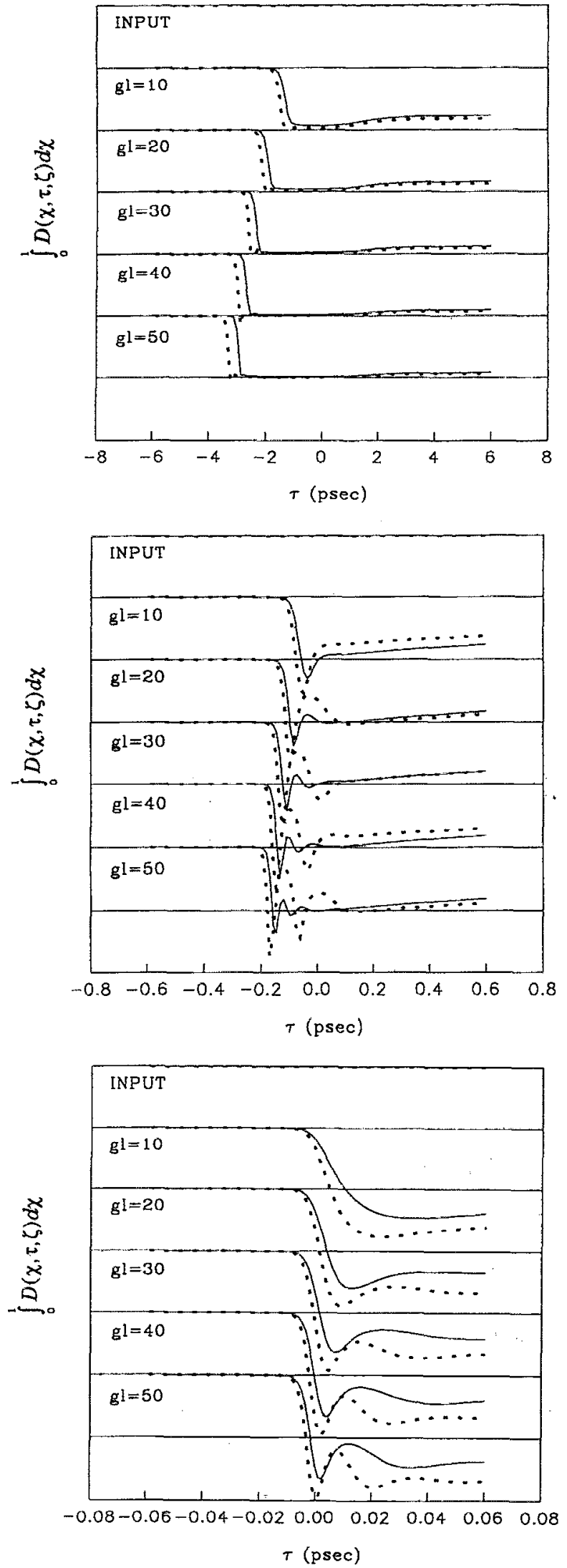

FIG. 8. Normalized integrated (over the entire solid angle) population differences as a function of time. Solid lines are the general semiclassical model (isotropic molecular distribution), and dotted lines are the unidirectional molecular distribution model. The input pulse widths are (a) $1 \times 10^{-12} \mathrm{~s},(\mathrm{~b}) 1 \times 10^{-13} \mathrm{~s}$, and (c) $1 \times 10^{-14} \mathrm{~s}$.

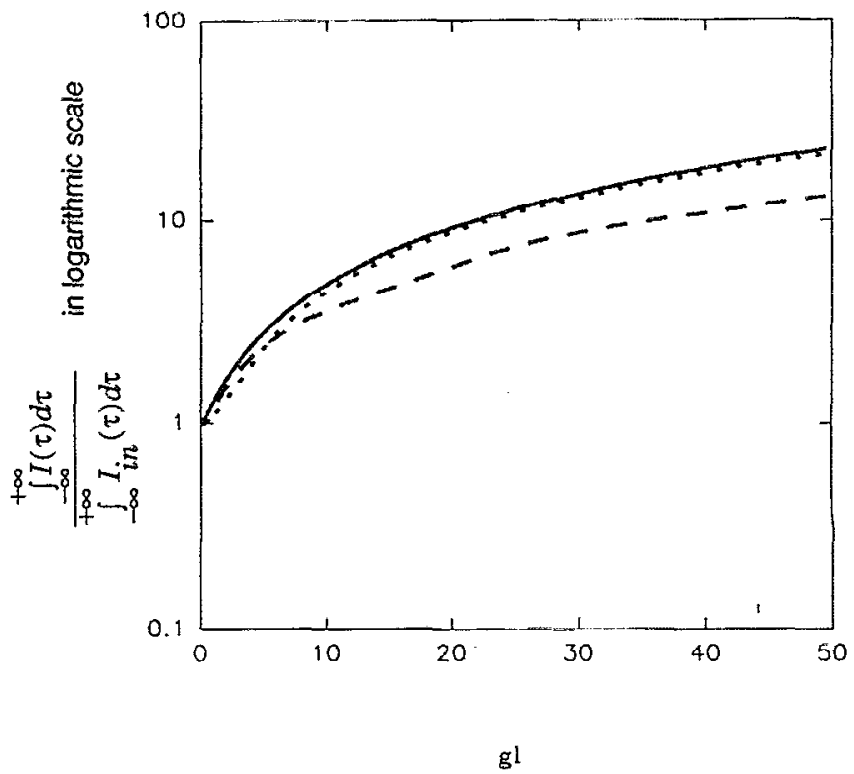

FIG. 9. Integrated pulse energy gain based on the unidirectional molecular distribution model of Eqs. (37) $-(40)$ as a function of small signal gain-length product for input pulse widths of $1 \times 10^{-12} \mathrm{~s}$ (solid lines), $1 \times 10^{-13} \mathrm{~s}$ (dashed lines), and $1 \times 10^{-14} \mathrm{~s}$ (dotted lines). Other parameters are the same as in Fig. 4.

oriented at an angle $\alpha$ with respect to the $x$ axis. Then the electric-field vectors and the unit dipole moment vectors can be written

$$
\begin{aligned}
& \mathbf{E}_{p}^{\prime}=E_{p}^{\prime}\left(\mathbf{e}_{x} \cos \alpha+\mathbf{e}_{z} \sin \alpha\right), \\
& \mathbf{E}_{s}^{\prime}=E_{s}^{\prime} \mathbf{e}_{x}, \\
& \mathbf{e}_{p}=\mathbf{e}_{s}=\mathbf{e}_{x} \cos \theta+\mathbf{e}_{y} \sin \theta \cos \phi+\mathbf{e}_{z} \sin \theta \sin \phi .
\end{aligned}
$$

The dot products of these vectors are

$\mathbf{E}_{p}^{\prime} \cdot \mathbf{e}_{p}=E_{p}^{\prime}\left(\mathbf{e}_{x} \cos \alpha+\mathbf{e}_{z} \sin \alpha\right) \cdot\left(\mathbf{e}_{x} \cos \theta+\mathbf{e}_{y} \sin \theta \cos \phi\right.$

$+\mathbf{e}_{z} \sin \theta \sin \phi$ )

$=E_{p}^{\prime}(\cos \alpha \cos \theta+\sin \alpha \sin \theta \sin \phi)$

$=E_{p}^{\prime}\left[\chi \cos \alpha+\left(1-\chi^{2}\right)^{1 / 2} \sin \alpha \sin \phi\right]$

$=E_{p}^{\prime} X$,

$\mathbf{E}_{s}^{\prime} \cdot \mathbf{e}_{s}=E_{s}^{\prime} \mathbf{e}_{x} \cdot\left(\mathbf{e}_{x} \cos \theta+\mathbf{e}_{y} \sin \theta \cos \phi+\mathbf{e}_{z} \sin \theta \sin \phi\right)$

$$
=E_{s}^{\prime} \chi,
$$

where the new angle variables are

$$
\begin{aligned}
& \chi=\cos \theta, \\
& X=\chi \cos \alpha+\left(1-\chi^{2}\right)^{1 / 2} \sin \alpha \sin \phi .
\end{aligned}
$$

It is evident that when the pump field is parallel to the signal field $(\alpha=0)$ the variables $X$ and $\chi$ are equal.

With the substitutions given in Eqs. (A4)-(A7), the simplified density-matrix equations (14)-(17) in Ref. 21 will have the same forms as for the longitudinal pumping case considered previously: 
$\frac{\partial \rho_{22}}{\partial t}=\frac{1}{\hbar} \eta_{s}^{\prime} E_{s}^{\prime} \chi-\frac{\rho_{22}}{\tau_{2}}+\frac{T_{p}\left|\mu_{p}\right|^{2}}{2 \hbar^{2}}\left(E_{p}^{\prime} X\right)^{2}$,

$\frac{\partial \rho_{11}}{\partial t}=-\frac{1}{\hbar} \eta_{s}^{\prime} E_{s}^{\prime} \chi-\frac{\rho_{11}}{\tau_{1}}+\frac{\rho_{22}}{\tau_{2}}$

$\frac{\partial \eta_{s}^{\prime}}{\partial t}=-\frac{1}{2 \hbar}\left(\rho_{22}-\rho_{11}\right)\left|\mu_{s}\right|^{2} E_{s}^{\prime} \chi-\frac{\eta_{s}^{\prime}}{T_{s}}$

$\frac{\partial E_{s}^{\prime}}{\partial z}+\frac{1}{v} \frac{\partial E_{s}^{\prime}}{\partial t}+\frac{\gamma_{s}}{2} E_{s}^{\prime}=-\frac{\mu \omega_{s}^{2}}{k_{s}} \frac{N}{2 \pi} \int_{0}^{1} \int_{0}^{2 \pi} \eta_{s}^{\prime} \chi d \chi d \phi$

except with $X$ taking the form of Eq. (A7).

Introducing the same normalization forms as those in Ref. 21 for the dependent variables and keeping in mind the new orientation factor $X$, one would have

$$
\begin{aligned}
D(\theta, z, t)= & \frac{\mu \omega_{s}^{2} N T_{s}\left|\mu_{s}\right|^{2}}{k_{s} \hbar \gamma_{s}} \\
& \times \frac{\chi^{2}\left(2-3 \sin ^{2} \alpha\right)+\sin ^{2} \alpha}{2 X^{2}}\left(\rho_{22}-\rho_{11}\right),
\end{aligned}
$$

$$
\begin{aligned}
M(\theta, z, t)= & \frac{\mu \omega_{s}^{2} N T_{s}\left|\mu_{s}\right|^{2}}{k_{s} \hbar \gamma_{s}} \\
& \times \frac{\chi^{2}\left(2-3 \sin ^{2} \alpha\right)+\sin ^{2} \alpha}{2 X^{2}}\left(\rho_{22}+\rho_{11}\right),
\end{aligned}
$$

$$
Q(\theta, z, t)=-\frac{\mu \omega_{s}^{2} N T_{s}\left|\mu_{s}\right|^{2}\left(2 \tau_{2} T_{s}\right)^{1 / 2}}{k_{s} \hbar \gamma_{s}}
$$

$$
\begin{gathered}
\times \frac{\chi^{2}\left(2-3 \sin ^{2} \alpha\right)+\sin ^{2} \alpha}{2 X^{2}} \eta_{s}^{\prime}, \\
A(z, t)=\left(\left|\mu_{s}\right| / \hbar\right)\left(\tau_{2} T / 2\right)^{1 / 2} E_{s}^{\prime} \\
P(z, t)=\frac{\mu \omega_{s}^{2} N T_{s}\left|\mu_{s}\right|^{2}}{k_{s} \hbar \gamma_{s}} \frac{\tau_{2} T_{p}\left|\mu_{p}\right|^{2} E_{p}^{\prime 2}}{2 \hbar^{2}} .
\end{gathered}
$$

With these normalization forms, one obtains the same differential equations for $D, M$, and $Q$ as given in Eqs. (1) - (3); and thus the only difference between longitudinal pumping and transverse pumping is the orientation factor $X$ which is now normalized away for these three variables:

$$
\begin{aligned}
\frac{\partial D}{\partial t}= & -\frac{1}{\tau_{2}}\left\{\left(1+\frac{\tau_{2}}{2 \tau_{1}}\right) D+\left(1-\frac{\tau_{2}}{2 \tau_{1}}\right) M+2 Q A \chi\right. \\
& \left.-P\left[\chi^{2}\left(1-\frac{3 \sin ^{2} \alpha}{2}\right)+\frac{\sin ^{2} \alpha}{2}\right]\right\}
\end{aligned}
$$

$$
\frac{\partial M}{\partial t}=-\frac{1}{\tau_{2}} \mid-\frac{\tau_{2}}{2 \tau_{1}} D+\frac{\tau_{2}}{2 \tau_{1}} M-P\left[\chi^{2}\left(1-\frac{3 \sin ^{2} \alpha}{2}\right)\right.
$$

$$
\left.\left.+\frac{\sin ^{2} \alpha}{2}\right]\right\}
$$

$$
\frac{\partial Q}{\partial t}=-\frac{1}{T_{s}}(Q-A D \chi)
$$

By substituting $E_{s}^{\prime}$ in terms of $A$, and $\eta_{s}^{\prime}$ in terms of $Q$ into Eq. (A11) and canceling all the common terms on both sides, one can obtain

$$
\frac{\partial A}{\partial z}+\frac{1}{v} \frac{\partial A}{\partial t}=-\frac{\gamma_{s}}{2} A+\frac{\gamma_{s}}{4 \pi} \int_{0}^{1} \int_{0}^{2 \pi} Q \chi \frac{X^{2}}{\chi^{2}\left(1-\frac{3}{2} \sin ^{2} \alpha\right)+\left(\sin ^{2} \alpha\right) / 2} d \phi d \chi
$$

Substituting Eq. (A7) into Eq. (A20), and carrying out the integral over $\phi$, one obtains

$$
\begin{aligned}
\frac{\partial A}{\partial z}+\frac{1}{v} \frac{\partial A}{\partial t} & =-\frac{\gamma_{s}}{2} A+\frac{\gamma_{s}}{4 \pi} \int_{0}^{1} \int_{0}^{2 \pi} Q \chi \frac{\left[\chi \cos \alpha+\left(1-\chi^{2}\right)^{1 / 2} \sin \alpha \sin \phi\right]^{2}}{\chi^{2}\left(1-\frac{3}{2} \sin ^{2} \alpha\right)+\left(\sin ^{2} \alpha\right) / 2} d \phi d \chi \\
& =-\frac{\gamma_{s}}{2} A+\frac{\gamma_{s}}{4 \pi} \int_{0}^{1} \int_{0}^{2 \pi} Q \chi \frac{\chi^{2} \cos ^{2} \alpha+\left(1-\chi^{2}\right) \sin ^{2} \alpha \sin ^{2} \phi+2 \chi \cos \alpha\left(1-\chi^{2}\right)^{1 / 2} \sin \alpha \sin \phi}{\chi^{2}\left(1-\frac{3}{2} \sin ^{2} \alpha\right)+\left(\sin ^{2} \alpha\right) / 2} d \phi d \chi \\
& =-\frac{\gamma_{s}}{2} A+\frac{\gamma_{s}}{4 \pi} \int_{0}^{1} \frac{Q \chi}{\chi^{2}\left(1-\frac{3}{2} \sin ^{2} \alpha\right)\left(\sin ^{2} \alpha\right) / 2} 2 \pi\left(\chi^{2} \cos ^{2} \alpha+\frac{\left(1-\chi^{2}\right) \sin ^{2} \alpha}{2}\right) d \chi \\
& =-\frac{\gamma_{s}}{2} A+\frac{\gamma_{s}}{2} \int_{0}^{1} \frac{Q \chi}{\chi^{2}\left(1-\frac{3}{2} \sin ^{2} \alpha\right)+\left(\sin ^{2} \alpha\right) / 2}\left(\chi^{2}\left(1-\sin ^{2} \alpha\right)+\frac{\left(1-\chi^{2}\right) \sin ^{2} \alpha}{2}\right) d \chi=-\frac{\gamma_{s}}{2}\left(A-S_{0}^{1} Q \chi d \chi\right),
\end{aligned}
$$


which is the same as Eq. (4). Thus, Eqs. (1)-(4) can be used for both longitudinally and transversely pumped dye laser amplifiers.

${ }^{1}$ W. H. Knox, IEEE J. Quantum Electron. QE-24, 388 (1988).

${ }^{2}$ E. P. Ippen and C. V. Shank, in Picosecond Phenomena, Springer Series in Chemical Physics, Vol. 4, edited by C. V. Shank, E. P. Ippen, and S. L. Shapiro (Springer, Berlin, 1978), p. 103.

${ }^{3}$ R. L. Fork, C. V. Shank, and R. Yen, Appl. Phys. Lett. 41, 223 (1982).

${ }^{4}$ A. Migus, A. Antonettí, J. Etchepare, D. Hulin, and A. Orszag, J. Opt. Soc. Am. B 2, 584 (1985).

${ }^{5}$ C. V. Shank, R. L. Fork, R. Yen, R. H. Stolen, and W: J. Tomlinson, Appl. Phys. Lett. 40, 761 (1982).

${ }^{6}$ T. L. Gustafson and D. M. Roberts, Opt. Commun. 43, 141 (1982).

${ }^{7}$ M. C. Downer, R. L. Fork, and M. Islam, in Ultrashort Phenomena IV, edited by D. H. Auston and K. B. Eisenthal (Springer, Berlin, 1984), p. 27.

${ }^{8}$ J. B. Hopkins and P. M. Rentzepis, Appl. Phys. Lett. 47, 776 (1985).

${ }^{9}$ C. Rolland and P. Corkum, Opt. Commun. 59, 64 (1986).

${ }^{10}$ D. S. Bethune, Appl. Opt. 20, 1897 (1981).
"T. L. Koch, L. C. Chiu, and A. Yariv, J. Appl. Phys. 53, 6047 (1982). ${ }^{12}$ A. Migus, J. L. Martin, R. Astier, and A. Orszag, in Picosecond Phenomena II, Springer Series in Chemical Physics, Vol. 14, edited by R. M. Hochstrasser, W. Kaiscr, and C. V. Shank (Springer, Berlin, 1980), p. 59.

${ }^{13}$ A. Migus, C. V. Shank, E. P. Ippen, and R. L. Fork, IEEE J. Quantum Electron. QE-18, 101 (1982).

${ }^{14}$ R. F. Leheny and J. Shah, IEEE J. Quantum Electron. QE-11, 70 (1983).

${ }^{15}$ U. Ganiel, A. Hardy, G. Neumann, and D. Treves, IEEE J. Quantum Electron. QE-11, 881 (1975).

${ }^{16}$ L. W. Casperson, J. Appl. Phys. 54, 2198 (1983).

${ }^{17}$ F. Kannari and M. Obara, J. Opt. Soc. Am. B 7, 1493 (1990).

${ }^{18}$ P. W. Milonni, R. B. Gibson, and A. J. Taylor, J. Opt. Soc. Am. B 5, 1360 (1988).

${ }^{19}$ K. E. Hill, G. H. C. New, and K. Burnett, J. Opt. Soc. Am. B 8, 839 (1991).

${ }^{20}$ F. Kannari, J. Appl. Phys. 67, 3954 (1990).

${ }^{21}$ S. H. Jiang and L. W. Casperson, J. Appl. Phys. 69, 1866 (1991).

${ }^{22}$ E. O. Schulz-Dubois, Bell Syst. Tech. J. 43, 625 (1964). 